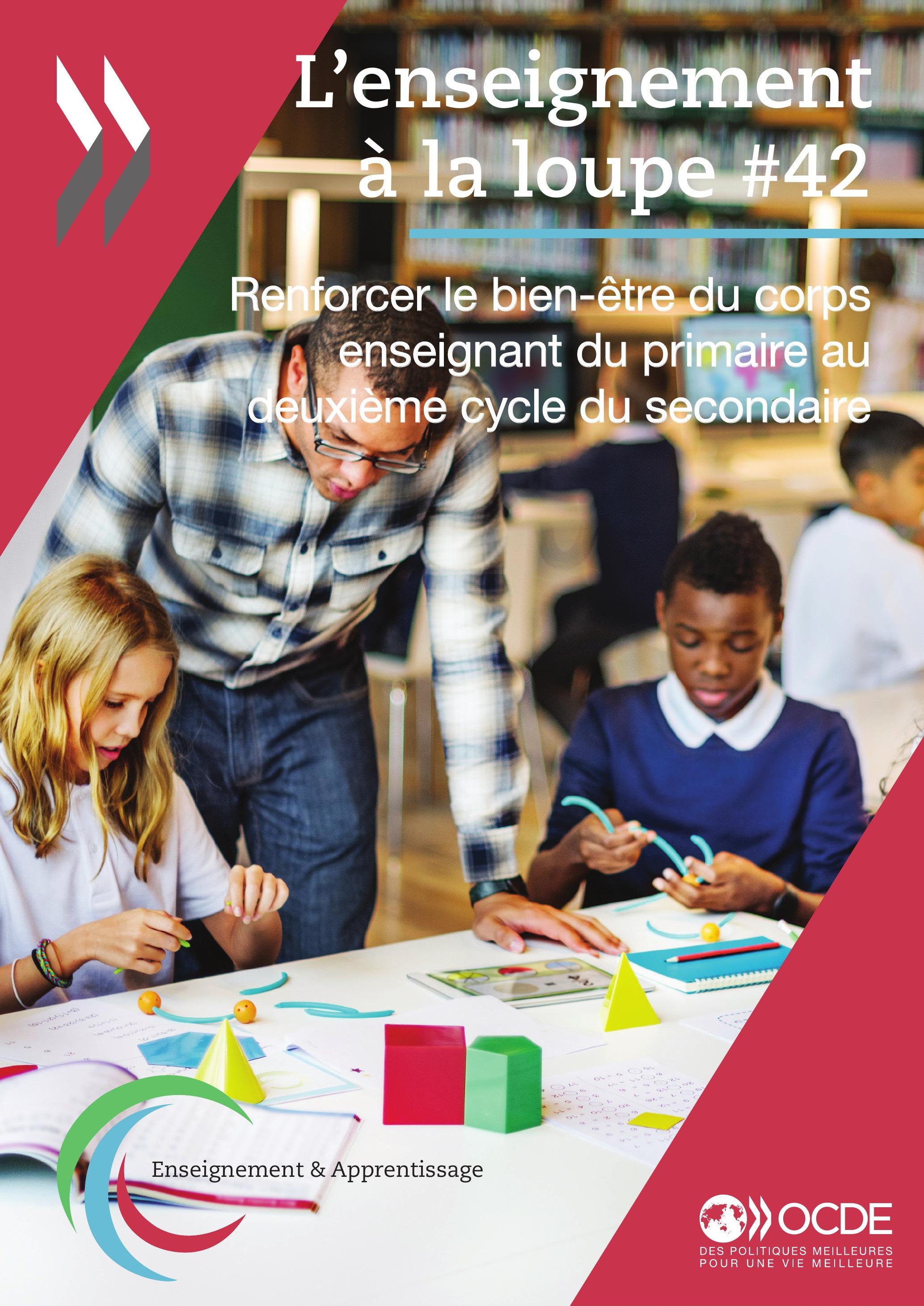




\section{Renforcer le bien-être du corps enseignant du primaire au deuxième cycle du secondaire}

- En moyenne, un enseignant sur six dans les établissements primaires et secondaires déclare ressentir « beaucoup » de stress dans le cadre de son travail. Or, les enseignants stressés sont plus susceptibles d'envisager de quitter la profession.

- La satisfaction professionnelle, le soutien de l'établissement et la motivation sont des facteurs essentiels pour réduire le stress au travail, car les enseignants satisfaits ont davantage tendance à vouloir rester dans la profession malgré un niveau de stress élevé.

- Les caractéristiques de l'établissement, tels qu'un climat propice, la collaboration et la possibilité de participer aux décisions de l'établissement, peuvent améliorer le bien-être des enseignants et minimiser les risques d'attrition.

À I'heure où les systèmes d'éducation sont confrontés au monde de l'après COVID-19, nous ne devons pas perdre de vue l'importance du bien-être des enseignants. Avant la pandémie, les enseignants éprouvaient déjà des difficultés face à la charge de travail et au stress, comme le montre l'Enquête internationale sur l'enseignement et l'apprentissage (TALIS), I'une des premières initiatives internationales visant à mesurer le bien-être du corps enseignant. Néanmoins, les établissements et les enseignants disposent des outils nécessaires pour améliorer le bien-être et réduire le stress sur le lieu de travail.

Ce document a pour objectif de donner un aperçu des actions concrètes que les établissements et les systèmes d'éducation pourraient mettre en œuvre pour améliorer le bien-être et la satisfaction professionnelle des enseignants.

\section{Qu'est-ce que l'Enquête TALIS ?}

Lancée en 2008, l'Enquête internationale sur l'enseignement et l'apprentissage (TALIS) est la première grande étude internationale auprès d'enseignants et de chefs d'établissement qui examine différents aspects ayant une incidence sur l'apprentissage des élèves. Elle donne une voix aux enseignants et aux chefs d'établissement, leur permettant de contribuer à l'analyse et au développement des politiques en éducation dans des domaines clés.

Les enseignants et les chefs d'établissement en poste dans le secteur public et privé dans le premier cycle de l'enseignement secondaire ordinaire constituent la population internationale cible de TALIS 2018. Dans chaque pays, un échantillon représentatif de 4000 enseignants a été prélevé de manière aléatoire dans 200 établissements, dont le chef d'établissement a aussi été sélectionné, en vue de l'enquête de 2018. Tous instruments d'enquête confondus, quelque 260000 enseignants représentatifs de plus de 8 millions d'enseignants dans les 48 pays et économies participants, ont été interrogés.

Les participants à l'enquête TALIS pouvaient opter pour la mise en œuvre de l'enquête dans les établissements du deuxième cycle de l'enseignement secondaire. Quinze pays et économies ont choisi d'administrer l'enquête TALIS dans l'enseignement primaire, et les données de l'enquête sont disponibles pour douze d'entre eux. Onze pays et économies ont choisi d'administrer l'enquête TALIS dans le deuxième cycle de l'enseignement secondaire, et des données sont disponibles pour dix d'entre eux.

La collecte de données a été réalisée entre septembre et décembre 2017 pour les participants de l'hémisphère sud, et entre mars et mai 2018 pour ceux de l'hémisphère nord. Les données ayant été collectées avant la crise de la COVID-19, on notera que certaines des fréquences et relations parmi les variables présentées ici peuvent avoir changé. Le site de l'enquête TALIS (www.oecd.org/education/talis) fournit de plus amples informations.

\section{Les établissements et les enseignants peuvent contribuer à réduire le stress}

Les données de l'enquête TALIS 2018 issues de l'enseignement primaire et secondaire avant la pandémie montrent qu'environ un enseignant sur six a déclaré ressentir beaucoup de stress au sein de son établissement. En moyenne, cela concerne $17 \%$ des enseignants du primaire pour 13 pays et économies, $18 \%$ des enseignants du premier cycle 
du secondaire pour 49 pays et économies et $15 \%$ des enseignants du deuxième cycle du secondaire pour 11 pays et économies.

Ces niveaux de stress déclarés par les enseignants ne doivent pas être négligés. Les analyses de régression sont révélatrices du phénomène de l'attrition. Elles montrent que les enseignants très stressés par leur travail sont plus susceptibles d'envisager de quitter l'enseignement dans les cinq ans dans la quasi-totalité des pays et économies participant à l'enquête TALIS, qu'il s'agisse de l'enseignement primaire, du premier ou du deuxième cycle de l'enseignement secondaire.

Cependant, les établissements et les enseignants sont en mesure de diminuer les effets du stress sur une éventuelle attrition. Les résultats de l'enquête TALIS pour le premier cycle du secondaire montrent que la satisfaction professionnelle, le soutien de l'établissement, la motivation et l'efficacité personnelle donnent une image quelque peu différente. La corrélation avec le stress n’est plus significative dans 18 pays et économies.

Graphique 1. Relation entre le stress des enseignants et l'intensité de leurs tâches

Probabilité d'envisager de quitter l'enseignement dans les cinq ans en lien avec le fait de souffrir de stress au travail « dans une grande mesure »

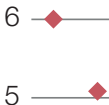

Rapport de cotes supérieur à 1 : Les enseignants déclarant souffrir de stress au travail « dans une grande mesure » sont plus susceptibles de quitter l'enseignement dans les cinq ans

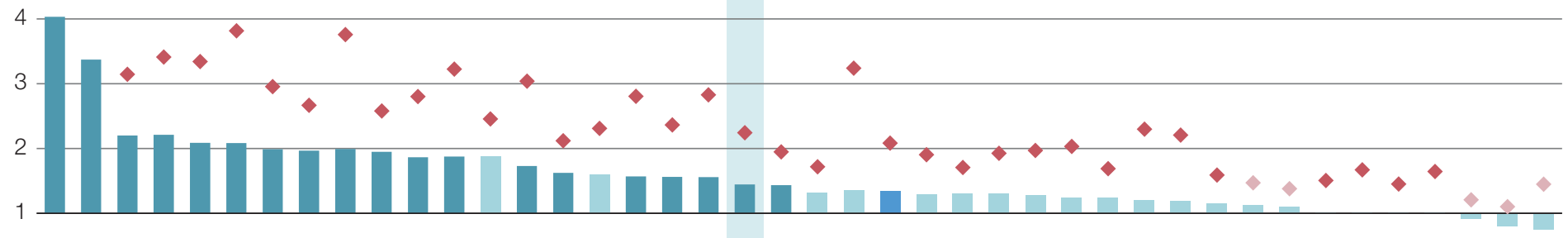

0

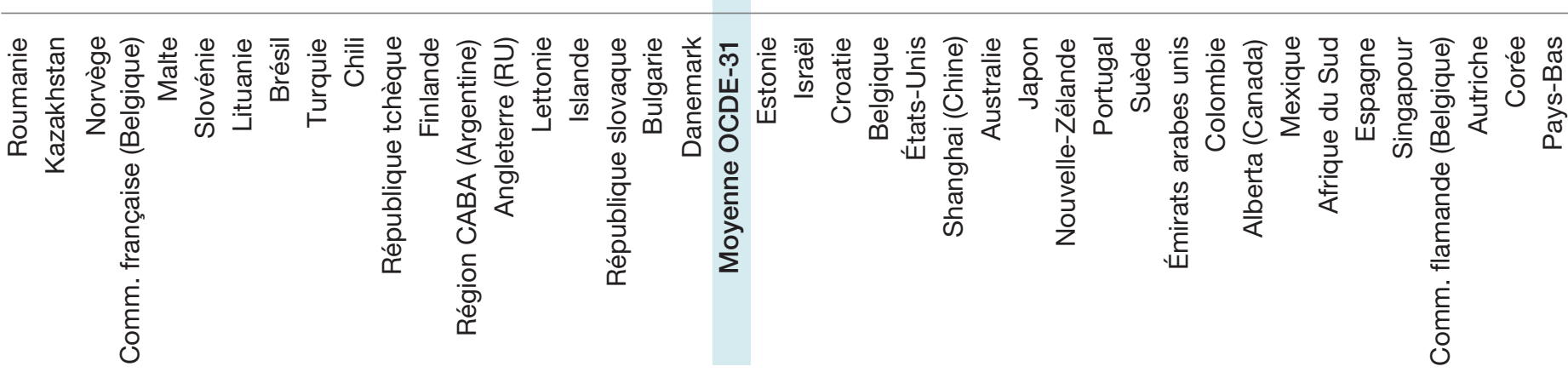

Remarques : Les valeurs statistiquement significatives sont indiquées dans un ton plus foncé

Résultats d'une régression logistique binaire basée sur les réponses des enseignants du premier cycle du secondaire.

Le rapport de cotes indique la mesure dans laquelle une variable explicative est associée à un résultat catégoriel. L'association est négative si le rapport de cotes est inférieur à 1 ; positive s'il est supérieur à 1 ; et nulle s'il est égal à 1.

La variable explicative est une variable binaire : la catégorie de référence correspond aux enseignants du premier cycle de l'enseignement secondaire souffrant de stress au travail " dans une grande mesure », " dans une certaine mesure », « pas du tout ».

L'analyse est limitée aux enseignants indiquant que leur enseignement dans la classe spécifique n'est pas directement ou essentiellement destiné à des élèves ayant des besoins spécifiques d'éducation. Ces données sont fournies par les enseignants et font référence à l'une de leurs classes actuelles, choisie au hasard dans leur emploi du temps hebdomadaire.

1. Après la prise en compte de l'âge, du sexe, de l'ancienneté dans l'établissement, du fait de travailler à temps plein et de la composition de la classe (pourcentage d'élèves peu performants, ayant des problèmes de comportement et issus de familles défavorisées sur le plan socioéconomique).

2. Après la prise en compte de l'âge, du sexe, de l'ancienneté dans l'établissement, du fait de travailler à temps plein et de la composition de la classe et de la satisfaction des enseignants concernant l'autonomie en classe, de la formation professionnelle, de la participation à des activités formelles d'initiation dans l'établissement, de la collaboration des enseignants dans les cours, de la perception des enseignants de la valorisation de leur profession dans la société, de l'efficacité personnelle et de la satisfaction professionnelle.

Les pays et économies sont classés par ordre décroissant de la probabilité des enseignants souffrant " dans une grande mesure » de stress professionnel, de quitter l'enseignement dans les cinq ans.

Source : OCDE (2020) Résultats de TALIS 2018 Nolume II) : Des Enseignants et Chefs d'Établissement comme Professionnels Valorisés, TALIS, OECD Publising, Paris https://doi.org/10.1787/69e92fca-fr, Tableaux II.2.68 et II.2.69, 
L'explication la plus plausible de cette différence de signification semble être l'introduction de l'échelle de satisfaction professionnelle. II existe en effet une corrélation négative significative entre l'intention des enseignants de quitter la profession dans les cinq ans et le niveau de satisfaction professionnelle dans 42 pays et économies ayant participé à l'enquête TALIS. De plus, les variables relatives au soutien de l'établissement, à la motivation et à l'efficacité personnelle semblent étroitement liées à la satisfaction professionnelle elle-même. On peut en déduire que, dans l'ensemble, parmi les pays ayant participé à l'enquête TALIS, notamment parmi les 18 pays pour lesquels le stress n'est plus significativement corrélé, la satisfaction au travail est un facteur majeur permettant de prédire le risque que les enseignants quittent l'enseignement, parfois indépendamment de leur niveau de stress.

Cela signifie donc que le stress et la satisfaction professionnelle jouent tous deux un rôle clé dans l'attrition des enseignants, avec quelques différences entre les pays et les économies ayant participé à l'enquête TALIS. La réduction du stress doit donc être prise au sérieux par les responsables politiques et les chefs d'établissement. Toutefois, l'importance que joue la satisfaction au travail donne à penser qu'il est possible d'atténuer les effets négatifs du stress, et de limiter ainsi l'attrition des enseignants.

\section{Améliorer le climat au sein de l'établissement pour retenir les enseignants}

Quelles actions concrètes les établissements peuvent-ils mettre en œuvre pour améliorer le bien-être des enseignants ? L'analyse des résultats de l'enquête TALIS montre que les enseignants qui travaillent dans des établissements où il existe une culture de collaboration caractérisée par un soutien mutuel ont tendance à déclarer un niveau de bien-être plus élevé. La pratique fréquente d'activités de collaboration entre collègues renforce aussi l'entretien de relations positives, consolide la confiance mutuelle, outre qu'elle soutient et améliore le climat global au sein de l'établissement. Ces aspects s'avèrent essentiels pour améliorer le bien-être des enseignants.

L'enquête TALIS montre que les enseignants du primaire qui travaillent dans des établissements où il existe une culture de collaboration caractérisée par un soutien mutuel sont moins susceptibles de vouloir quitter l'enseignement dans les cinq ans. De même, les enseignants du deuxième cycle du secondaire qui ont la possibilité de participer aux décisions de l'établissement sont également moins susceptibles de vouloir quitter la profession (graphique 2).

Ces résultats donnent à penser que les mesures de soutien mises en œuvre par les établissements et dont l'objectif est d'atténuer le stress à l'échelle de l'établissement, de la circonscription et du pays amélioreront le bien-être du corps enseignant. Les enseignants bénéficieront d'une aide supplémentaire pour exercer leurs fonctions ainsi que d'un espace où leurs besoins et leurs difficultés seront entendus. La collaboration et le soutien de l'établissement sont essentiels pour renforcer la résilience des enseignants et les préparer au changement. 


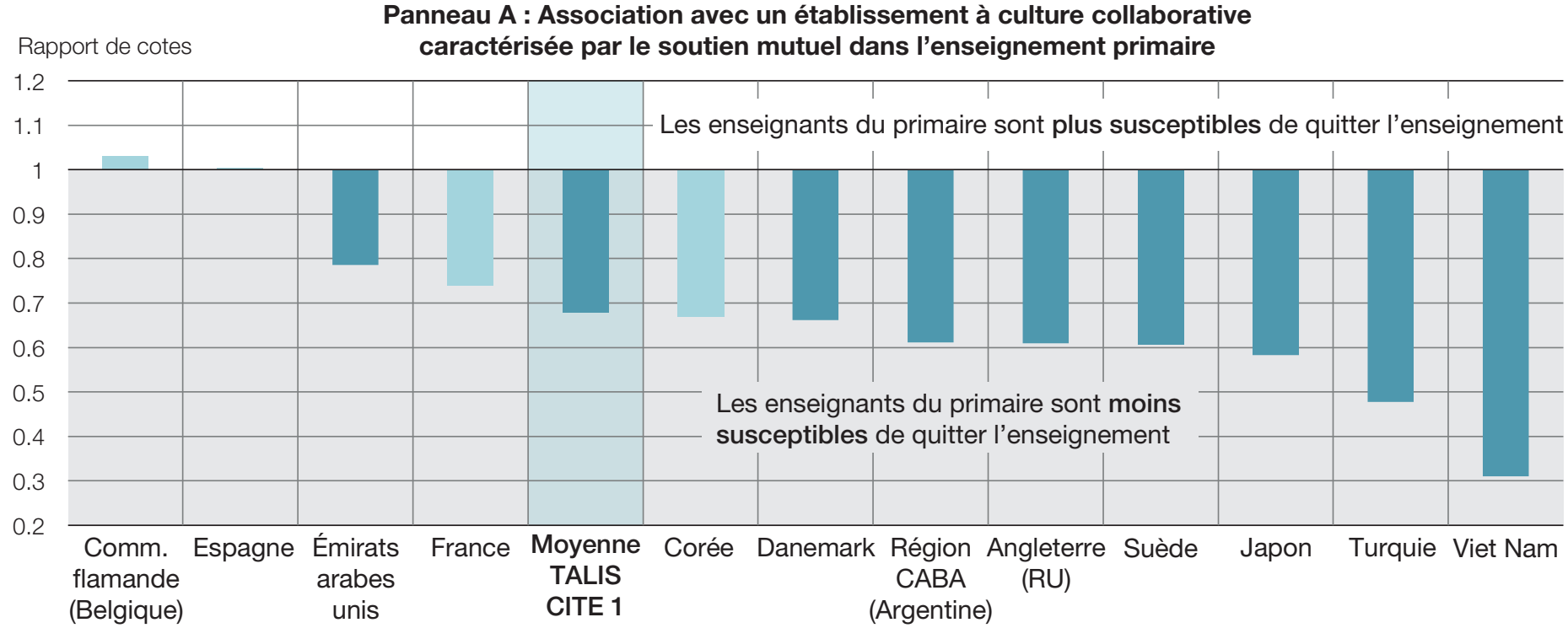

Panneau B : Association avec un établissement offrant aux membres du personnel la possibilité de participer Rapport de cotes activement aux décisions le concernant dans le deuxième cycle de l'enseignement secondaire

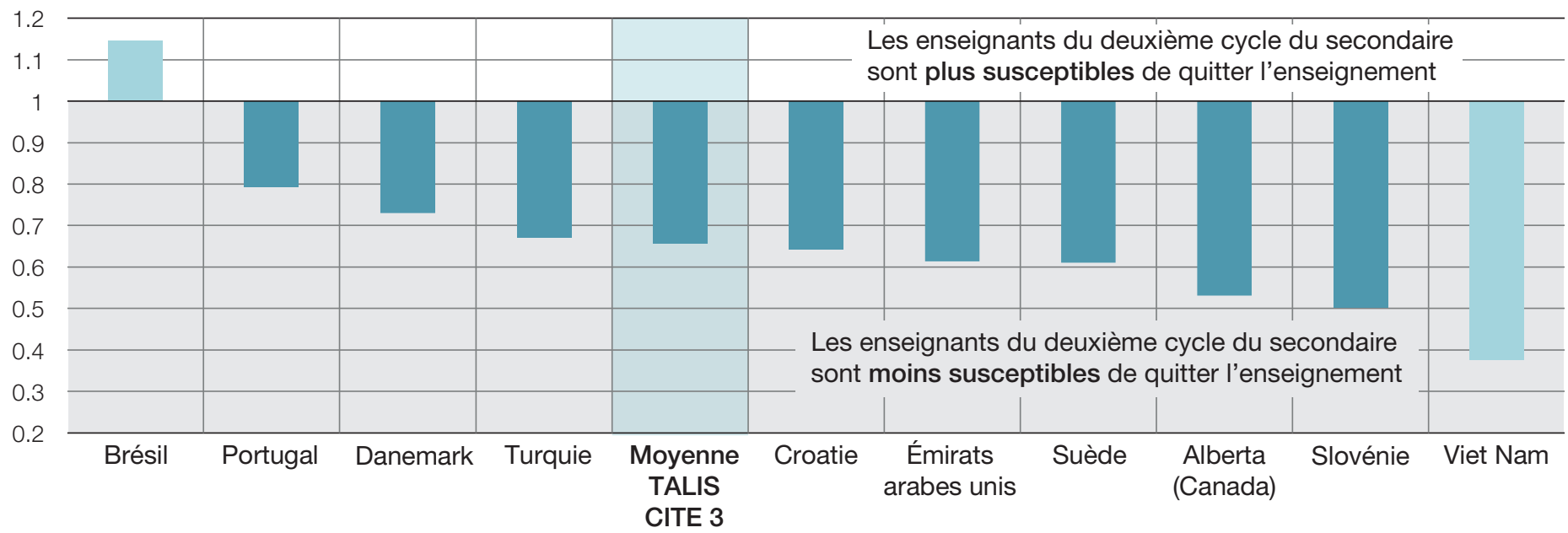

Remarque : Les valeurs statistiquement significatives sont indiquées dans un ton plus foncé.

Les pays et les économies sont classés par ordre décroissant de la probabilité que les enseignants quittent la profession dans les cinq ans lorsqu'il existe une culture de collaboration au sein de l'établissement caractérisée par un soutien mutuel dans l'enseignement primaire (panneau A) et lorsque l'établissement offre au personnel la possibilité de participer aux décisions le concernant dans le deuxième cycle de l'enseignement secondaire (panneau B).

Source : OCDE (2021), Teachers Getting the Best out of Their Students: From Primary to Upper Secondary Education, TALIS, Éditions OCDE, Paris,

https://doi.org/10.1787/5bc5cd4e-en, tableaux 6.37 et 6.38 (publication en français à paraître).

\section{Pour conclure}

Le soutien et l'amélioration du bien-être des enseignants devraient être au cœur des systèmes d'éducation, à l'heure où les établissements essaient de trouver leurs marques dans le monde de l'après COVID-19. La pandémie a amené avec elle de nouvelles difficultés auxquelles sont confrontés les enseignants. Ils ont dû par exemple s'adapter à des environnements d'apprentissage en ligne. Outre leurs responsabilités habituelles, ils ont dû préparer des cours pour l'apprentissage à distance, motiver leurs élèves et les évaluer à distance. II n'est pas surprenant que ces nouvelles modalités de travail aient exacerbé la tendance des enseignants à vouloir quitter le métier.

Les données de l'enquête TALIS 2018 antérieures à la pandémie montrent que plus le stress ressenti par les professionnels de l'éducation est important, plus la probabilité qu'ils quittent la profession est élevée. Mais elles indiquent également que l'attrition liée au stress peut être atténuée en augmentant la satisfaction professionnelle. 
Si les conclusions de l'enquête montrent que les caractéristiques des établissements aident les enseignants à relever les défis de leur vie professionnelle, les politiques adoptées dans les établissements peuvent également contribuer à développer cette résilience. Cela signifie que les chefs d'établissement doivent contribuer activement à instaurer au sein de leur établissement un climat scolaire et des conditions de travail plus propices. Les pouvoirs publics pourraient aussi accorder plus d'importance à la responsabilisation collective qu'à la responsabilisation individuelle, et faire en sorte que les enseignants jouissent d'une plus grande autonomie pour veiller au respect des normes de leur propre travail. De même, responsables politiques et chefs d'établissement pourraient créer les espaces et les ressources nécessaires au développement des pratiques collaboratives. Toutefois, la collaboration repose aussi sur la bonne volonté des enseignants qui doivent mobiliser leurs connaissances, se faire confiance et se soutenir les uns les autres pour assurer leur bien-être sur le long terme.

\section{Consulter}

\section{www.oecd.org/education/talis/}

\section{Personnes à contacter}

Pablo Fraser (pablo.fraser@oecd.org)1 et talis@oecd.org.

\section{Remarque}

1. Nous tenons à remercier Aakriti Kalra, ancienne consultante externe, et Asuka Ohagi, analyste à l'OCDE, pour leur contribution à la préparation de ce numéro de L'enseignement à la loupe.

\section{Pour en savoir plus}

OCDE (2020), Résultats de TALIS 2018 (Volume II) : Des Enseignants et Chefs d'Établissement comme Professionnels Valorisés, TALIS, Éditions OCDE, Paris, https://doi.org/10.1787/69e92fca-fr.

OCDE (2021), Teachers Getting the Best out of Their Students: From Primary to Upper Secondary Education, TALIS, Éditions OCDE, Paris, https://doi.org/10.1787/5bc5cd4e-en (publication en français à paraître).

La publication de ce document relève de la responsabilité du secrétaire général de l'OCDE. Les opinions et les interprétations exprimées ne reflètent pas nécessairement les vues des pays membres de l'OCDE.

Ce document, ainsi que les données et cartes qu'il peut comprendre, sont sans préjudice du statut de tout territoire, de la souveraineté s'exerçant sur ce dentier, du tracé des frontières et limites internationales, et du nom de tout territoire, ville ou région.

Les données statistiques concernant Israël sont fournies par et sous la responsabilité des autorités israéliennes compétentes. L'utilisation de ces données par l'OCDE est sans préjudice du statut des hauteurs du Golan, de Jérusalem-Est et des colonies de peuplement israéliennes en Cisjordanie aux termes du droit international.

Vous êtes autorisés à copier, télécharger ou imprimer du contenu OCDE pour votre utilisation personnelle. Vous pouvez inclure des extraits des publications, des bases de données et produits multimédias de l'OCDE dans vos documents, présentations, blogs, sites internet et matériel d'enseignement, sous réserve de faire mention de la source OCDE et du copyright. Les demandes pour usage public ou commercial ou de traduction devront être adressées à rights@oecd.org.

Crédits photo : Première de couverture @ Rawpixel ; quatrième de couverture ( ) Rawpixel, @ wavebreakmedia, @ Rawpixel

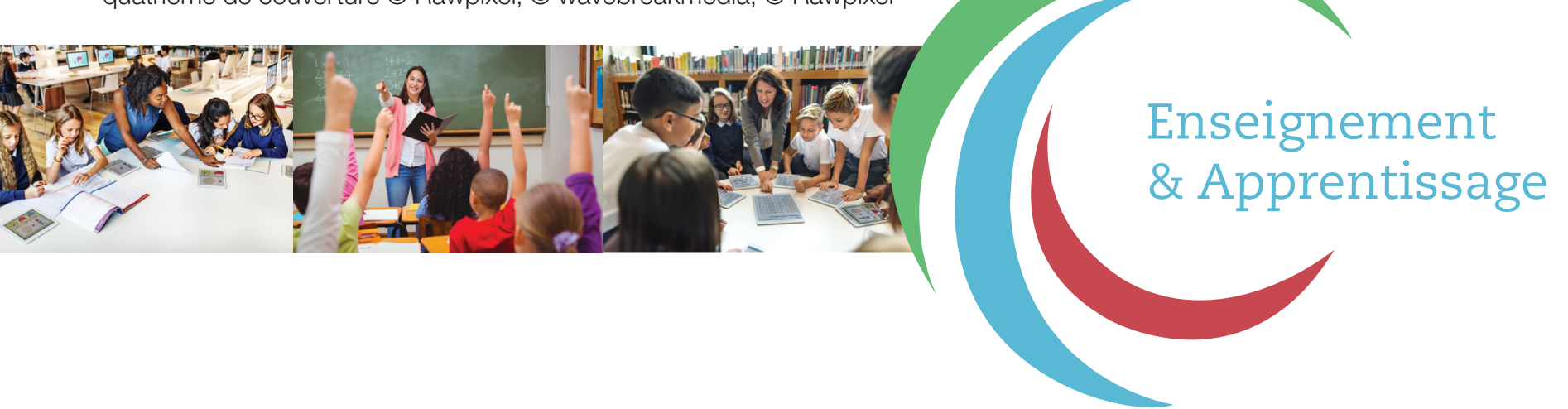

